\title{
SOBRE UNA NUEVA Y DESAIRADA TRADUCCIÓN DE WITTGENSTEIN
}

Ludwig Wittgenstein, Investigaciones filosóficas, traducción de Jesús Padilla Gálvez, Editorial Trotta, Madrid 2017, 327 páginas.

Esta breve nota sólo tiene la pretensión de indicar algunas de las características de esta nueva edición en español de la obra de Wittgenstein y tomar posición crítica frente a ciertas decisiones del traductor o errores de la versión.

En español la obra de Ludwig Wittgenstein, Investigaciones filosóficas, sólo ha tenido la versión de Alfonso García Suárez y Ulises Moulines, publicada por la Unam en 1986 y reeditada en conjunto por Crítica o sólo por esta editorial en distintas ocasiones posteriores. Dada la importancia del texto y el hecho de que la edición de García y Moulines (G\&M) sea de referencia en nuestro ámbito, en la valoración de esta nueva traducción de Padilla Gálvez (PG) será inevitable tener presente aquella primera versión, como, por lo demás, lo hace el propio PG. Éste, en efecto, tras la mención de los datos de la primera edición en español, en las primeras palabras que dirige al trabajo de G\&M lo asocia a las deficiencias de la versión inglesa de Elizabeth Anscombe, sobre la cual se habría basado el trabajo de aquellos: «Dichas dificultades se trasladan claramente a la traducción española» (18) ${ }^{1}$. Ni una palabra de reconocimiento a los méritos y a la importancia ya histórica que en nuestro medio ha tenido el trabajo pionero de G\&M. Sin mediar declaración de guerra, PG va a lo suyo: «Los traductores transcriben en parte del inglés los errores y las faltas cometidas en la edición original. Pero también se toman considerables licencias que no aparecen en el texto original alemán: palabras intercambiadas, conjugaciones mal traducidas» (18 s.) y pasa a dar un ejemplo canónico de ello. Para dar una idea del enfoque de PG, quizás valga la pena entrar con algún detalle en este primer ejemplo del error detectado en G\&M.

En el $§ 85$ se lee en G\&M: "Así

que traduce la oración en alemán:

«Also kann ich sagen, der Wegweiser läßt doch keinen Zweifel offen».

Ahora bien, PG señala que «en el texto original del archivo de Cambridge,

1 Los números entre paréntesis corresponden a la edición comentada. 
Wittgenstein escribe y corrige:

“Also kann ich sagen, der Wegweiser läßt doch (k)einen Zweifel offen”.» (19),

cuya versión, por supuesto, es exactamente la contraria a la de G\&M:

«Así pues, puedo decir que el indicador de caminos deja una duda abierta».

Se trata, como es evidente, de una corrección editorial que no puede hacerse sino con base en la colación de manuscritos, pero de ningún modo a partir de una mera interpretación del texto, como pretende indicarlo PG, con un inocultable aire de suficiencia: «Cualquier especialista se hubiera dado cuenta [etc.]»(19). Pues sí, el lector cuidadoso del texto se encuentra aquí con una cierta dificultad, pero si eso es lo que dice el texto alemán, caben posibilidades de interpretación dentro del idiosincrático y zigzagueante hilo de pensamiento de Wittgenstein en las Investigaciones. Si hay que hacer una corrección al texto alemán, ello es responsabilidad del editor, no del traductor en cuanto intérprete, por eso queda mal que ahora, con todas las ayudas editoriales a disposición, que no existían hace treinta años, este nuevo traductor quiera cargarle la mano a los primeros traductores que, en este punto, hicieron bien su trabajo, pues haber procedido con base en su interpretación del pasaje habría sido irresponsable como oficio de traducción. Por cierto, PG no menciona que el texto en alemán de la editorial Suhrkamp, al cuidado de Joachim Schulte, publicado en 1984 y que en 1999 iba en la duodécima edición, mantiene la versión keinen (288), lo que viene a confirmar, por si hiciera falta, que la corrección einen por keinen no era procedente antes del pesado trabajo editorial que se realizó en el último decenio sobre las distintas versiones manuscritas de las Investigaciones. G\&M aquí nunca cometieron un error y que PG lo presente como tal ya deja de ser un mero error.

Esta discusión pone de presente, por cierto, una de las principales características de esta nueva traducción, cual es el haber podido recurrir a ediciones y manuscritos que sólo en los últimos diez o quince años han estado a disposición de los investigadores y que han servido de base para la nueva ola de traducciones de Wittgenstein. A lo largo de su trabajo, el traductor PG tiene cuidado de hacer mención de las precisiones que aportan los distintos manuscritos y que quedan recogidas en su versión. Por esta razón, el texto de PG será de consulta durante los próximos años, mientras no se haga una difusión asequible de los materiales en los que se basa su versión. Para el estudioso de lengua española queda claro ahora cuán compleja es la construcción de las Investigaciones filosóficas de Wittgenstein, con sus varias versiones, sus correcciones internas, sus vacilaciones, repeticiones y supresiones. En este sentido, dicho trabajo es un buen indicador del cuidado con el que hay que proceder en la investigación wittgensteiniana contemporánea, que tras los grandes estudios del pasado siglo ha arribado en éste a un nuevo nivel de sutileza y detalle que abre nuevas avenidas para la comprensión del pensamiento del filósofo austríaco.

Empero, lo que habría podido ser el mayor aporte del trabajo de PG se queda en una precaria ayuda al no tratarse de una edición bilingüe alemán-español, como ha sido costumbre inveterada en las ediciones de las obras mayores de Wittgenstein. Desde ya hay que decir que, pese a sus anotaciones de detalle y de filiación de los manuscritos, esta edición de la editorial Trotta de ningún modo va a remplazar a la 
edición de la editorial Crítica, por la sencilla razón de que para el lector de lengua española que cuenta con conocimientos de alemán no hay simplemente otra edición de las Investigaciones filosóficas donde pueda encontrarse el texto alemán original encarado página por página. Las circunstancias de ahorro o la imposibilidad de negociar con la editorial Blackwell o un criterio editorial discutible, sitúan de entrada esta versión en notable condición de inferioridad frente a la anterior. En cierto sentido, para el trabajo cotidiano bastaría con tomar nota de algunos de los aportes de PG frente a G\&M y seguir utilizando esta última. Eso sí, hay que apresurarse a anotar que no todas las decisiones de traducción de PG representan una mejora de G\&M; algunas de ellas, en realidad, son un retroceso, mientras que en otras simplemente repite los errores de la primera.

El traductor PG ofrece sus principales decisiones de traducción en una enrevesada Introducción —-sobre la que se volverá más adelante—, donde disputa a la vez y a la par con la versión inglesa de Anscombe y con la española de G\&M, como si esta última fuera casi una versión del inglés y no del alemán. Algunas de sus observaciones son justas, si bien su modo de presentación podría ser menos contencioso y más comedido. Un acierto en relación con G\&M es la de verter Vorstellung como representación y no por imagen, mientras que Bild se vierte por imagen y no por figura, como hacen G\&M. Esta es quizás la diferencia de más nota y donde PG mejora notablemente a G\&M, haciendo la lectura no sólo más fiel al pensamiento del austríaco, sino también más aprehensible intuitivamente.

Pueden mencionarse otros aspectos donde PG representa una mejoría efectiva respecto de G\&M. Traducir en §29c auffassen por concebir y no por interpretar. Traducir en \$40 sprachwidrig por ilícitamente en el lenguaje y no sólo por ilícitamente. Utilizar el recurso a la tabla de colores en $\S 48$ (75), donde G\&M simplemente traen indicados los colores con abreviaturas de los nombres (67). Corregir en $§ 68 \mathrm{~b}$ la traducción de "Es ist nicht überall von Regeln begrenzt», que G\&M daban erróneamente como: «No está en absoluto delimitado por reglas" (89), con el "No está delimitado por doquier mediante reglas» (85), que si bien no es muy feliz, al menos es correcta. Traducir en $\$ 89 \mathrm{c}$ besinnen por reflexionar y no por acordarse. Traducir en $§ 103$ Lebensluft por aire para vivir y no sólo por aire; aunque quizás habría sido mejor aire de vida, recordando la forma de vida de \$23, Lebensform. Traducir en $\S 185 \mathrm{~d}$ von Natur por con naturalidad, en lugar del anterior por naturaleza. Traducir en $\$ 318$ wir denkend sprechen por hablamos pensando y no por pensamos mientras hablamos. Traducir en $\$ 466$ tatsächlich por efectivamente y no por realmente. Hay otros lugares donde PG implica una mejoría de G\&M, no tanto como tema de traducción, sino por claridad o simplemente por errores de levantamiento del texto en G\&M. Pero también hay numerosos pasajes donde PG reitera algunas dificultades de G\&M, y otros, donde ha introducido las suyas propias. Como observación general puede hacerse notar que son pocos los lugares, relativamente hablando, donde PG ha significado una diferencia cualitativa respecto de G\&M.

Ahora bien, una decisión mayor, problemática en español, hay que reconocerlo, pero a la que PG no le ha dado una solución satisfactoria, es la traducción de meinen o Meinung, donde PG se ha dejado llevar por la idea de que «a Wittgenstein le juegan una mala pasada los anglicismos adquiridos a raíz de sus largas estancias en Inglaterra» (27); es decir, que influido por el inglés, en este caso por el verbo to mean y el sustantivo meaning, Wittgenstein mismo ya no sabría usar los términos 
alemanes correspondientes meinen y Meinung. Por eso, corrigiendo no sólo a Anscombe y a G\&M, sino al propio Wittgenstein, PG no se sonroja al afirmar que «en el contexto del uso específico que hace Wittgenstein hemos optado por lo general por traducirlo [meinen] como "referir"» (28). ¿Es esto cierto?, es decir, ¿que PG ha vertido meinen como referir? ¿No lo habrá traducido, más bien, como referirse? ¿Y es referirse una versión apropiada de meinen, mejor, en todo caso, que la de significar de G\&M? Pueden revisarse algunos de los pasajes correspondientes para ver qué tan acertada ha sido esta decisión de PG. Así, del cumplimiento de una orden se dice en $\S 186$ :

«Der richtige Schritt ist der, welcher mit dem Befehl — wie er gemeint war- übereinstimmt»,

que en PG deviene:

«El paso correcto es el que concuerda con la orden — tal como fue referida» (§186).

La expresión en alemán es clara, el proceder correcto no consiste sólo en seguir la orden tal cual, sino también lo que ella quiere decir, su sentido. Tómese el ejemplo del propio Wittgenstein:

\footnotetext{
«Alguien me dice: “¿Enséñales un juego a los niños!”. Yo les enseño a jugar dinero a los dados y el otro me dice: "No me refería a un juego así”» ( $\$ 69$, nota).
}

La expresión alemana «Ich habe nicht so ein Spiel gemeint» puede entenderse también como «No era un juego así lo que yo quería decir». Este sentido de la expresión alemana gemeint no queda recogido, ni siquiera aludido en la opción de PG de referida.

No, por supuesto, hay un error en todos los casos donde PG utiliza referirse (no referir, como erróneamente indica) por meinen; muchos son acertados. Lo que no se entiende es que en lugar de mantener abierta la perspectiva interpretativa, haya formulado la solemne y vacua declaración de que ni Anscombe ni G\&M, y tampoco Wittgenstein mismo, conocen el verdadero significado de meinen como referir, que será la versión que él adopta, "por lo general». Así rápidamente puede verse que en $\S 142$ nota, $\S 156 \mathrm{c}, \S 183$ y $\S 677$, PG, con buen sentido, ha visto que no podía aferrarse a su referir y ha debido optar por el más castizo de querer decir. El caso de $\$ 677$ es emblemático, pues la versión irrumpe en medio de la sección que va entre $§ 661$ y $§ 693$, donde el tema es justamente el de meinen, esto es, querer decir, que en algún caso al menos, en $\$ 669$ y en $\$ 684$, Wittgenstein distingue del referir en sentido propio, beziehen. Ciertamente, a raíz de esta decisión extrema, PG habrá de terminar ofreciendo el español referencia como traducción tanto de Meinung, $\S 186$, como de Bezug, §10b, sin que el lector se aperciba de las diferencias.

Ilustrativo es también el caso de $§ 183$, donde Wittgenstein presenta tres condiciones en las cuales alguien dice "Puedo andar», "Ich kann gehen»; estas condiciones son "tengo tiempo», "ya estoy lo suficientemente fuerte» $\mathrm{y}$ «en lo que respecta al estado de mis piernas». Pero en la enunciación de la segunda condición, PG comete un error de atención, dice: "Puedo nadar, es decir, ya estoy bastante fuerte» (130). ¿Puedo nadar? Se dirá que es posible la confusión entre 'andar' y 'nadar'. Sí, sin duda es posible. Pero, ¿qué tan posible es cometer exactamente el mismo error que ya habían cometido en el mismo pasaje G\&M? En efecto, allí se lee: «Puedo nadar, o sea, ya estoy bastante fuerte» (185). Parece que PG, que acusa a G\&M de plegarse a Anscombe, no ha podido tampoco dejar de mirar a G\&M. 
Con igual irrelevancia PG ha declarado que en G\&M «tampoco se fue muy coherente en la traducción de Gebrauch, Verwendung y Anwendung. Hemos traducido Gebrauch por "uso", Verwendung por "uso" o "empleo", y Anwendung por "aplicación”" (25). Sin embargo, y sin ninguna pretensión exhaustiva, puede verse que en $\$ 84$ Anwendung se traduce por 'uso' (92), igual que en el pasaje al que se refiere $\S 84$, que es $\S 68$ b (85). En ambos casos, G\&M han traducido por 'aplicación', pero PG los ha criticado por su falta de coherencia.

Otra anotación de PG, igual de presuntuosa, si no más, se encuentra en §168. Hablando del acto de leer, Wittgenstein dice que «Auch gleitet der Blick [...] über die gedruckte Zeile», esto es, "también la mirada se desliza [...] sobre la línea impresa», donde G\&M ponen «el ojo se desliza», lo que le da a PG ocasión de sacar a relucir el arsenal de su malignidad: "Wittgenstein diferencia claramente mirada (Blick) y ojo (Auge); la versión anterior los traducía indistintamente, lo que genera cierta confusión» (124, n.121). ¿De verdad genera "cierta confusión»? Seguro alguien supone que un ojo físico va deslizándose sobre la página impresa. No que G\&M sea correcto, pues recurre a una figura donde no era necesario hacerlo, pero es claro, al menos, que se trata de una figura. PG tendría que haber corregido y seguir en silencio. Eso sí, por supuesto, PG ni se percata de que en este respecto él ha cometido un error mayor que aquel que le achaca a «la versión anterior». En efecto, en $§ 156 c$ Wittgenstein ya ha utilizado esta imagen de la mirada que se desliza sobre la página, así:

\footnotetext{
«Was geht nun vor sich, wenn er, z. B., die Zeitung liest? —Seine Augen gleiten —wie wir sagen- den gedruckten Wörtern entlang».

«¿Qué ocurre, entonces, cuando, por ejemplo, él lee el periódico? — Sus ojos se deslizan —como decimos- a lo largo de las palabras impresas».
}

¿Qué ha dicho, empero, PG?

"¿Qué sucede, pues, cuando, por ejemplo, lee el periódico? —Sus ojos siguen — como decimos-difusamente las palabras impresas» (118).

Por supuesto, con esta versión de gleiten como seguir, PG destroza la unidad de argumentación wittgensteiniana respecto del acto de leer, pues en este punto no es lícito disociar la correlación entre $§ 156 \mathrm{c}$ y $§ 168$. Por cierto, el propio Wittgenstein es quien ha dado pie para la versión de G\&M de ojo en vez de mirada; no es que esté bien, pero ello muestra que la nota de PG (124, n.121) no sólo es improcedente, sino falsa en este punto: aquí Wittgenstein no establece una distinción conceptual entre las dos expresiones "el ojo se desliza sobre la página impresa» y "la mirada se desliza sobre la página impresa». El énfasis no está allí, sino en la acción de deslizarse. Por cierto, la versión de PG no sólo cambia el deslizarse por seguir, sino que le añade un difusamente, que no está en el texto en alemán y que, por supuesto, arroja una niebla difusa sobre la comprensión del texto wittgensteiniano.

Un caso difícil, de particular relevancia, consiste en la traducción de Verständigung. Aquí PG ha querido tomar distancia de la versión más usual, correcta también, de comunicación, y con su actitud habitual menciona que esta palabra significa «entendimiento, acuerdo o reconciliación» (53, n.6). Así, cuando Wittgenstein dice en $\S 3$ que «Augustinus beschreibt, könnten wir sagen, ein System der Verständigung», PG ofrece: "Podríamos decir que Agustín de Hipona describe un sistema de entendimiento» (53) — frente al más claro de G\&M, «sistema de 
comunicación»-, con lo que hace casi ininteligible lo que Wittgenstein allí afirma. De nuevo, con las incongruencias que le son propias, PG había traducido en §2, «Die Sprache soll der Verständigung eines Bauenden A mit einem Gehilfen B dienen», como «El lenguaje debe servir en la comunicación entre un albañil A con su aprendiz B». No es erróneo en §22, §143 y §242 traducir Verständigung como comprensión, como lo hacen PG y G\&M, pero hay que mantener a la vista la acepción de comunicación, correcta también, y que ayuda a la mejor aprehensión del texto wittgensteiniano.

Particularmente chocante es la traducción de §95, «Man kann denken, was nicht der Fall ist», como «Se puede pensar lo que no es un acontecimiento» (98), en lugar de G\&M: «Se puede pensar lo que no es el caso» (117), que recoge el eco tractariano, tan importante en las Investigaciones.

Desorientadora es la traducción de $\$ 100$, «es ist doch dann verunreinigt, und ich interessiere mich nun für dasjenige, was hier verunreinigt wurde», como «después de todo está enrarecido y yo me intereso pues por lo que aquí ha sido enrarecido» (100), donde el verbo verunreinigen no puede sino significar ensuciar, contaminar (Duden, 1672), y expresa la convicción de las Investigaciones de huir del suelo cristalino del Tractatus. Increíble y lamentablemente, en este punto G\&M también tienen un desliz grave: "Está contaminado y yo me intereso por lo que está limpio" (119), en lugar del correcto "Sí, está contaminado, y yo me intereso ahora por aquello que aquí se contaminó».

Nótese así mismo la deficiente versión de §108a, «Die Betrachtung muß gedreht werden, aber um unser eigentliches Bedürfnis als Angelpunkt», como «Hay que darle la vuelta a la observación, pero para que nuestra necesidad real sea la piedra angular» (101), donde el eje, Angelpunkt, alrededor del cual, um, debe hacerse girar, muß gedreht werden, la consideración, Betrachtung, se convierte en «la piedra angular», lo que nada tiene que ver con la acción de girar y sí sugiere, de paso, un fundamentalismo ajeno al espíritu wittgensteiniano.

Una de las oraciones emblemáticas del pensamiento de Wittgenstein, aparece en §109, «Die Philosophie ist ein Kampf gegen die Verhexung unsres Verstandes durch die Mittel unserer Sprache», que PG vierte como "La filosofía es una lucha contra el embrujo de nuestro entendimiento mediante los procesos de nuestro lenguaje» (102), tomando distancia del canónico de G\&M: «La filosofía es una lucha contra el embrujo de nuestro entendimiento por medio de nuestro lenguaje» (123). Aquí la dificultad, por supuesto, se encuentra en la expresión die Mittel, literalmente los medios, como en "poner a disposición los medios para un proyecto» (Duden, 1025), que en español, igual que en alemán, puede significar los recursos. Por eso, y a pesar de la pérdida de impacto retórico, quizás sea más apropiado mantener el plural con su sugerencia del dinero como medios, como recursos, entre otras cosas porque, al igual que el significado, el dinero puede tanto convertirse en un fetiche, como simplemente usarse por el valor que tiene (§120). Así, quizás sea preferible una traducción literal, con la ambigüedad que conlleva de si los medios contribuyen al embrujo o a la lucha contra el embrujo: «La filosofía es una lucha contra el embrujo de nuestro entendimiento por los medios de nuestro lenguaje».

Una dificultad particular la ofrece §125e: «Die bürgerliche Stellung des Widerspruchs, oder seine Stellung in der bürgerlichen Welt: das ist das philosophische Problem», que PG vierte como «La posición que asume la contradicción en la so- 
ciedad burguesa, o su posición en el mundo burgués: ese es el problema filosófico» (106). Aquí el texto recibe un énfasis histórico-político, que el adjetivo bürgerlich permite en su segunda acepción, como referencia a Bürgertum, del francés bourgeoisie, burguesía, mientras que la primera acepción hace alusión a lo propio de la sociedad civil (Duden, 295), que G\&M han sabido recoger: «El estado civil de la contradicción, o su estado en el mundo civil: ése es el problema filosófico» (129), insinuando también una oposición con el mundo militar y uniforme de la lógica.

Nótense los varios errores que se cometen en la traducción de §153:

«Wir versuchen nun, den seelischen Vorgang des Verstehens, der sich, scheint es, hinter jenen gröbern und uns daher in die Augen fallenden Begleiterscheinungen versteckt, zu erfassen. Aber das gelingt nicht. Oder, richtiger gesagt: es kommt gar nicht zu einem wirklichen Versuch».

PG dice:

«Tratamos, pues, de abarcar el proceso mental de la comprensión que parece ocultarse tras esos fenómenos concomitantes que nos llaman la atención y por ello más visibles. Pero no da resultado. O dicho más correctamente: no alcanza a ser un intento real» (117).

\section{Compárese con G\&M:}

«Tratamos, pues, de apresar el proceso mental de entender que parece ocultarse tras esos fenómenos concomitantes más gruesos y por ello más visibles. Pero no da resultado. O dicho más correctamente: no alcanza en absoluto a ser un intento real» (153).

Quizás la traducción más apropiada aquí del verbo erfassen sea comprender o aprehender, aunque el apresar de G\&M también es correcto y preferible al abarcar de PG. Pero este no es el problema. Lo grave es que PG no ha traducido gröbern, que quizás mejor que gruesos, podría ser burdos, groseros, y lo ha transliterado como aquello que nos llama la atención, que es inaceptable. Y más adelante simplemente ha omitido la expresión gar nicht, en absoluto. Dos errores serios en cuatro líneas.

La situación de §293b requiere de un comentario particular. Se trata del famoso ejemplo de las cajas. Cada uno tiene una caja, pero nadie puede mirar en la caja del otro y uno dice que en su caja hay algo que se llama «escarabajo» y que lo sabe sólo por mirar dentro de su caja. La argumentación concluye con la siguiente expresión: «Nein, durch dieses Ding in der Schachtel kann "gekürzt werden"; es hebt sich weg, was immer es ist». PG se compromete con esta versión: "No, mediante esa cosa en la caja se puede "recortar"; se suprime, sea lo que fuere» (158). No esperará el traductor que alguien entienda qué quiere decir su expresión. ¿De qué se está hablando? El verbo kürzen aquí hace alusión a la simplificación de un quebrado (Duden, 915); por ejemplo, si se tiene $6 / 8$, que equivale a $3 \times 2 / 4 \times 2$, este puede simplificarse como 3/4, es decir, «der Bruch kann gekürzt werden durch 2», «el quebrado puede simplificarse por $2 »$, que en el ejemplo no es más sino simplificar el contenido de las cajas y suprimir de todas ellas ese «escarabajo» que las hace redundantes. La traducción sería: «No, puede simplificarse por esa cosa que hay en la caja; se cancela, sea lo que sea».

Lo que ocurre en $\$ 402 \mathrm{a}$ es notoriamente inadmisible. Dice el texto: «die Vorstellungswelt ist ganz in der Beschreibung der Vorstellung dargestellt». Sin inmutarse, PG propone: "la descripción de la representación está completamente representada en el mundo de la representación» (182). No se trata de una traducción particularmente difícil, salvo quizás por la presencia en la misma oración de las palabras cercanas Vorstellung y darstellen. Puede ofrecerse sin más esta versión: 
«el mundo de la representación está representado por completo en la descripción de la representación». Aquí, para distinguir la representación de Vorstellung del representar de darstellen se le ha puesto una cursiva a esta última, "representar», siguiendo la misma sugerencia de PG para Darstellung (22). PG ha invertido la relación de los términos de la oración y ha puesto «la descripción está representada en el mundo», cuando el texto dice lo contrario: «el mundo está representado en la descripción».

Muchas otras inexactitudes y ligerezas pueden encontrarse con una simple inspección del texto, pero para concluir se hará breve mención de cuatro puntos de particular relevancia, a saber, el juego de lenguaje, la traducción de Agustín, el Índice de materias, y el carácter de la Introducción.

La famosa expresión de las Investigaciones, en §23, «Das Wort "Sprachspiel” soll hier hervorheben, daß das Sprechen der Sprache ein Teil ist einer Tätigkeit, oder einer Lebensform», se encuentra en PG del siguiente modo: «La expresión "juego de lenguaje" debe realzar aquí que el hablar la lengua es parte de una actividad o una forma de vida» (62), donde verter con dos palabras distintas, lenguaje y lengua, una misma palabra alemana, Sprache, que en su primera aparición se encuentra compuesta con Spiel, juego, es introducir una sutileza, a saber, la distinción entre lenguaje y lengua, que no se encuentra en el original. Así mismo, haber dejado de mencionar la repetición de la preposición de en «de una actividad o una forma de vida» podría dar a entender que hablar el lenguaje es ya una forma de vida y no una parte de ella, como es inequívoco en el original con el genitivo einer. Sin ser plenamente satisfactoria, la versión canónica de G\&M evita ambas ambigüedades: "La expresión "juego de lenguaje" debe poner de relieve aquí que hablar el lenguaje forma parte de una actividad o de una forma de vida» (39).

En relación con las diez menciones del nombre «Augustinus» en las Investigaciones filosóficas, hay que hacer notar que PG siempre lo presenta como "Agustín de Hipona», dándole una solemnidad al texto y un tufillo de intelectualidad que está ausente de la obra de Wittgenstein, que se halla muy familiarizado con escritos del obispo de Hipona, en particular con las Confesiones, como lo sabe cualquier lector del austríaco. Así mismo, respecto de los pasajes de Agustín de los que Wittgenstein no ofrece una versión en alemán, PG tendría que haber recurrido a alguna de las muchas y excelentes traducciones disponibles del Hiponense y no arriesgar su propia versión. Tal es el caso, en particular, en §436b, donde Wittgenstein cita a Agustín, Confesiones, 11.22.28: «Manifestissima et usitatissima sunt, et eadem rursus nimis latent, et nova est inventio eorum», de lo que PG ofrece su propia y deplorable traducción: «Estas cosas son las más utilizadas y las más claras de todas, y, sin embargo siguen estando demasiado ocultas (detrás) y el descubrimiento es siempre nuevo" (190, n.194). La traducción de referencia de la editorial Gredos, a cargo de Alfredo Encuentra Ortega, que PG cita en la Bibliografía (39), pero de la que no hace uso, propone esta excelente versión: «Son cosas evidentísimas y repetidísimas y, al mismo tiempo, estas mismas se hallan muy ocultas, y es novedoso su descubrimiento» (569).

Hay que indicar, sin más preámbulos, que el Índice de materias ha sido tomado de la edición de Hacker \& Schulte (H\&S) en la cuarta edición de Philosophical Investigations (2009), lo cual, de suyo, no es un error, aunque ignorar el buen Î́ndice alfabético de G\&M no ha sido un proceder afortunado. El Índice, en general, es 
utilizable, pero el lector de esta obra, enteramente en español, va a quedar un poco desconcertado cuando se encuentre la entrada de cada término no sólo en su lengua sino también en alemán. El recurso puede ser útil, pero parece improcedente en este caso. Empero, ese no es el problema con este Índice. Lo que ocurre con él es que fue confeccionado prácticamente por el procedimiento de «copy / paste», con los ajustes impuestos por la traducción literal en español, pero sin tener en cuenta las opciones y las decisiones de la traducción. Unas cuantas referencias a instancias ya tratadas permitirán aclarar el punto — los números son de parágrafos-.

Tómese el caso de aplicación, Anwendung: como ya se mencionó, PG no es coherente en la versión de este término, aunque hace aspavientos de ello frente a G\&M. El Índice alemán dice: "Anwendung —eines Wortes, 84, 340» (268), que obedientemente PG ha recogido como: «aplicación (Anwendung) — de una palabra (eines Wortes): 84, 340» (308). Como ya se dijo, en §84, PG ha traducido: «Dije del uso de una palabra», donde uso está por Anwendung. ¿Es entonces pertinente la entrada para el término aplicación en este Índice?

Tómese el caso de referir, meinen. El Índice alemán trae: «meinen —kein geistiges Handeln, kein Vorgang, keine Erfahrung, 19, 20, 35, 60, [etc.]» (279), que de nuevo, PG toma a la letra, así: "referir(se) (meinen) — sin acción mental, sin proceso, sin experiencia (kein geistiges Handeln, kein Vorgang, keine Erfahrung): 19, 20, 35, 60, [etc.]»(323). Pero su versión de $\$ 60$ no habla de referir ni de referirse; el pasaje pertinente reza así: "¿quién dice que la escoba está en el rincón quiere realmente decir: el palo y el cepillo están allí [etc.]?»(82), que corresponde al alemán: "Also meint der, der sagt, der Besen stehe in der Ecke, eigentlich: der Stiel sei dort und die Bürste [etc.]?»(33s). Ni una palabra en español de meinen como referir, pues, como es obvio, contra sus declaraciones dogmáticas, PG ha tenido que traducir aquí meinen por querer decir. El Índice de nuevo es aquí inútil y falso. Por supuesto que su traducción tiene otro problema, que cambia el sentido y hace que su texto sea en extremo confuso, pues debería ser: «quien dice que la escoba está en el rincón, ¿quiere realmente decir: el palo y el cepillo están allí [etc.]?».

Un tercer caso servirá de prueba de que errores de este tipo son recurrentes en el Índice. Ya se indicó que Verständigung puede tener varios equivalentes en español, dependiendo del contexto y del énfasis que el traductor quiera darle. La cuestión aquí es que PG nunca dice que Verständigung puede verterse como comunicación, al contrario, de un modo pomposo y vacuo, en $\$ 3$ propone entendimiento en un contexto que no aguanta esta palabra, como ya se mencionó. El Índice alemán, por supuesto, simplemente recoge las apariciones del término, así: «Verständigung, 2, 3, 22, 143, 242» (284); en el Índice de PG, empero, la palabra aparece como: «comprensión (Verständigung): 2, 3, 22, 143, 242»(310). Una rápida inspección muestra que en §2, Verständigung se ha traducido como comunicación, la comunicación de un albañil con su ayudante (52); en §3, es el ininteligible «sistema de entendimiento» (53); ahora, que en §22 sea comprensión (62), igual que en $\$ 143$ (113) y §242 (145), no elimina el doble error de esta entrada.

Otras ligerezas del Índice podrían aducirse, pero hay que insistir en la descomedida actitud de PG hacia G\&M. Si le hubiera lanzado tan sólo una ojeada al Índice de estos últimos, se habría encontrado con la notoria entrada de «chichones» (534), que lo habría salvado de la ridícula traducción de §119: «Los resultados de la filosofía son el descubrimiento de algún que otro simple sinsentido y prominencias 
que el entendimiento se ha hecho al chocar con los límites del lenguaje» (104). El término prominencias vierte Beulen que, por supuesto, se halla en el Índice alemán (270), pero que será inútil buscar en el Índice de PG, que ha eliminado de paso la que quizás sea la única aparición de los chichones en la literatura filosófica.

Hay que mencionar, por último, que la Introducción de PG se basa ampliamente en la de H\&S, ya aludida. Esto se muestra, primero, en observaciones centrales que PG simplemente ha tomado de H\&S, como las siguientes:

«Some of the substantive changes we have introduced into the translation are systematic. Anscombe had a marked preference for minimizing the use of the third-person impersonal pronoun “one" [etc.]» (H\&S, xiii);

"Algunos de los cambios característicos que hemos introducido en la traducción son sistemáticos. Anscombe tenía una marcada preferencia por reducir al mínimo el uso de la tercera persona del pronombre impersonal "uno" [etc.]» (PG, 24).

"Some German words that Wittgenstein employs are problematic for any translator. So, for example, his use of Satz has no obvious English equivalent [etc.]» (H\&S, xiv);

«Algunas palabras alemanas que Wittgenstein emplea son problemáticas para cualquier traductor. Así, por ejemplo, el uso de Satz no tiene equivalente obvio en español [etc.]» (PG, 25).

«Similar recurrent difficulties arise with the translation of Seele, since it cannot always be correctly rendered by "soul" [etc.]» (H\&S, xiv);

"Ciertas dificultades recurrentes surgen con la traducción del término Seele, ya que no siempre puede ser trasladado correctamente por "alma" [etc.]» (PG, 26).

"In the case of Empfindung the German noun has a much wider application than the English "sensation" [etc.]», (H\&S, 15);

«En el caso de Empfindung, el sustantivo alemán tiene un uso mucho más extenso que la palabra castellana "sensación" [etc.]" (PG, 26).

«Three recurrent errors run through Anscombe's translation. First, she commonly mistranslated manch(er, -e, -es) [...] Second, she apparently misunderstood the usage of wohl [...] Finally, there are occasions where the use of the German definite article der (die, das) should not be translated by a definite, but by an indefinite article [etc.]» (H\&S, xvi);

«La traducción de Anscombe comete tres errores recurrentes que repercuten en la primera edición castellana. Tienen que ver con el uso del pronombre indefinido manch(er, -e, -es) [...] En segundo lugar, se ha entendido mal el uso del adverbio wohl [...] Por último, hay ocasiones en las que el uso del artículo definido del alemán der (die, das) no permite un uso unívoco en castellano. Lo mismo ocurre con el artículo indefinido, etc. [etc.]» (PG, 28).

Pueden añadirse más ejemplos, pero la última mención basta para indicar la que quizás sea la confusión más seria que atraviesa el texto de PG. Para explicarla, hay que hacerse cargo del carácter de la edición inglesa de H\&S, que no se presenta como una nueva traducción, sino como una mejora y puesta al día de la benemérita traducción de Anscombe. Ahora bien, como PG sigue de cerca los pasos de H\&S - no que no haya propuesto mejoras de su propia cosecha-, pero, a diferencia de ellos, sí hace explícita su pretensión de ofrecer una nueva traducción, se encuentra en la inestable posición de quien tendría que reconocer los méritos de la traducción anterior, que ésta tan sólo pretende mejorar — que es lo que hacen H\&S con Anscombe-, pero que no puede reconocérselos, porque, a diferencia de ellos, está ofreciendo una nueva traducción. De allí que PG no se haya tomado en realidad el trabajo de confrontarse con la anterior versión de G\&M, sino que simplemente la asimiló a Anscombe y la descartó junto con ésta. Pero mientras que H\&S no descartan a Anscombe, PG sí descarta a G\&M, a quienes maltrata sin el menor pudor. Empero, hay un detalle de su trabajo que PG no logra resolver, cual es que no pudo disponer para la publicación del nuevo texto alemán de H\&S, por lo que mientras éstos hablan no sólo como traductores, sino sobre todo como editores, PG debe limitarse desde sus notas a lanzar apreciaciones editoriales sin contexto y sin fondo. Por cierto, nótese que PG, en la última traducción del inglés 
de H\&S - aunque él nunca dice que esté traduciendo de allí-, propone «el uso del artículo definido del alemán der (die, das) no permite un uso unívoco en castellano. Lo mismo ocurre con el artículo indefinido, etc.» como versión de "the use of the German definite article der (die, das) should not be translated by a definite, but by an indefinite article», en una increíble falta de comprensión del texto inglés. Tristemente, lagunas como éstas no se encuentran, ni mucho menos, ausentes del todo en esta nueva traducción de las Investigaciones filosóficas de Ludwig Wittgenstein.

BiBLIOGRAFÍA

PG

Ludwig, W. (2017). Investigaciones filosóficas, Traducción, introducción y notas críticas de Jesús Padilla Gálvez. Madrid: Editorial Trotta.

G\&M

Ludwig, W. (1988). Investigaciones filosóficas, Traducción de Alfonso García Suárez y Ulises Moulines. México-Barcelona: Unam-Crítica.

$\mathrm{H} \& \mathrm{~S}$

Ludwig, W. (2009). Philosophische Untersuchungen / Philosophical Investigations, Traducción de G. E. M. Anscombe, P. M. S. Hacker \& Joachim Schulte, Blackwell. Oxford: Malden MA..

Ludwig, W. (1984). Tractatus logico-philosophicus / Tagebücher 1914-1916 / Philosophische Untersuchungen, Suhrkamp, Fráncfort a. M., 121999.

San Agustín (2010. Confesiones, Traducción de Alfredo Encuentra Ortega. Madrid: Gredos. Duden (1989). Deutsches Universalwörterbuch, 2a ed. Mannheim: Dudenverlag. 